\title{
Transcatheter Mitral Valve Replacement: Update on New Devices
}

\author{
Matthew J. Gillespie, MD ${ }^{1,2^{*}}$, Robert C. Gorman, MD ${ }^{2,3}$, Joseph H. Gorman III, MD ${ }^{2,3}$ \\ ${ }^{1}$ The Children's Hospital of Philadelphia, Division of Cardiology, Philadelphia, PA, USA \\ ${ }^{2}$ Gorman Cardiovascular Research Group, Department of Surgery, Philadelphia, PA, USA \\ ${ }^{3}$ Perelman School of Medicine, University of Pennsylvania, Philadelphia, PA, USA
}

\begin{abstract}
Transcatheter Mitral valve replacement represents the next frontier in cardiac valve therapy. This review article details the authors' experience thus far in the development of a novel catheter-based mitral valve replacement device, and also highlights the preliminary experiences of other new technologies.

Copyright (c) 2016 Science International Corp.
\end{abstract}

\section{Key Words}

Mitral valve replacement - Mitral regurgitation • Transcatheter valve replacement • Ischemic mitral regurgitation • General

\section{Conflict of Interest}

The authors each have ownership interest in AnnuIon LLC, which is a company formed around this and other mitral valve technologies

\section{Comment on this Article or Ask a Question}

\section{Suggested Reading}

1. De Backer O, Piazza N, Banai S et al. Percutaneous transcatheter mitral valve replacement: An overview of devices in preclinical and early clinical evaluation. Circ Cardiovasc Interv 2014;7:400-409.

2. Herrmann HC, Maisano F. Transcatheter therapy of mitral regurgitation. Circulation
2014;130:1712-1722.

3. Karimov JH, Massiello AL, Fukamachi K. Overview of current sutureless and transcatheter mitral valve replacement technology. Expert review of medical devices 2013;10:73-83.

4. Shuto T, Kondo N, Dori Y et al. Percuta- neous transvenous melody valve-in-ring procedure for mitral valve replacement. J Am Coll Cardiol 2011;58:2475-2480.

5. Kondo N, Shuto T, McGarvey JR et al. Melody valve-in-ring procedure for $\mathrm{mi}-$ tral valve replacement: Feasibility in four annuloplasty types. Ann Thorac Surg
Fax +1 2037853346

E-Mail: jshd@scienceinternational.org http://structuralheartdisease.org/ (c) 2016 Journal of Structural Heart Disease Published by Science International Corp. ISSN 2326-4004

Accessible online at: http://structuralheartdisease.org/
* Corresponding Author: Matthew J. Gillespie, MD The Children's Hospital of Philadelphia Department of Cardiology

3401 Civic Center Blvd., Philadelphia, PA 19104, USA

Tel. +1 215590 1790; Fax: +1 215590 5415; E-Mail: gillespie@email.chop.edu 
2012;93:783-788.

6. Gillespie MJ, Minakawa M, Morita M et al. Sutureless mitral valve replacement: Initial steps toward a percutaneous procedure. Ann Thorac Surg 2013;96:670-674.

7. Attmann $T$, Pokorny $S$, Lozonschi $L$ et al. Mitral valved stent implantation: An overview. Minim Invasive Ther Allied Technol 2011;20:78-84.

8. Cheung A, Webb JG, Wong DR et al. Transapical transcatheter mitral valve-in-valve implantation in a human. Ann Thorac Surg 2009;87:e18-20.

9. Banai $\mathrm{S}$, Jolicoeur $E M$, Schwartz $\mathrm{M}$ et al. Tiara: A novel catheter-based mitral valve bioprosthesis: Initial experiments and shortterm pre-clinical results. J Am Coll Cardiol 2012;60:1430-1431.

10. Bapat V, Buellesfeld L, Peterson MD et al. Transcatheter mitral valve implantation (tmvi) using the edwards fortis device. Eurolntervention 2014;10 Suppl U:U120-128.

11. Cheung A, Webb J, Verheye $S$ et al. Short- term results of transapical transcatheter mitral valve implantation for mitral regurgitation. J Am Coll Cardiol 2014;64:1814-1819.

12. Piazza N, Bolling $\mathrm{S}$, Moat $\mathrm{N}$, Treede $\mathrm{H}$. Medtronic transcatheter mitral valve replacement. Eurolntervention 2014;10 Suppl U:U112-114.

13. Anyanwu AC, Adams DH. Transcatheter mitral valve replacement: The next revolution? J Am Coll Cardiol 2014;64: 18201824.

14. Hasan R, Mahadevan VS, Schneider $H$, Clarke B. First in human transapical implantation of an inverted transcatheter aortic valve prosthesis to treat native mitral valve stenosis. Circulation 2013;128:e74-76.

15. Sinning JM, Mellert $F$, Schiller W, Welz A, Nickenig G, Hammerstingl C. Transcatheter mitral valve replacement using a balloon-expandable prosthesis in a patient with calcified native mitral valve stenosis. European heart journal 2013;34:2609.
16. Fassa AA, Himbert D, Brochet $E$ et al. Emergency transseptal transcatheter mitral valve-in-valve implantation. Eurolntervention 2013;9:636-642.

17. Fassa AA, Himbert $D$, Brochet $E$ et al. Transseptal transcatheter mitral valve implantation for severely calcified mitral stenosis. JACC Cardiovascular interventions 2014;7:696-697.

18. Lozonschi L, Quaden R, Edwards NM, Cremer J, Lutter G. Transapical mitral valved stent implantation. Ann Thorac Surg 2008;86:745-748.

Cite this article as: Gillespie MJ, Gorman RC, Gorman JH. Transcatheter Mitral Valve Replacement: Update on New Devices. Structural Heart Disease 2016;2(2): 44-45. DOI: http://dx.doi.org/ 10.12945/j.jshd.2016.015.14 